\title{
La interrelación de diferentes disciplinas para el abordaje científico de fenómenos y problemas sociales
}

The interrelation of different disciplines for the scientific approach of phenomena and social problems

\author{
Winter Edgar Reyna Cruz \\ Centro de Investigaciones Económicas, Administrativas y Sociales - \\ Instituto Politécnico Nacional (CIECAS - IPN), México \\ psicologo.winter.reyna@gmail.com \\ Al redo Leonardo Carreón Corona \\ Centro de Investigaciones Económicas, Administrativas y Sociales - \\ Instituto Politécnico Nacional (CIECAS - IPN), México \\ alfredocarreon93@hotmail.com \\ Silverio Gerardo Armijo Mena \\ Centro de Investigaciones Económicas, Administrativas y Sociales - \\ Instituto Politécnico Nacional (CIECAS -IPN), México \\ sgerardo@cinvestav.mx
}

\section{RESUMEN:}

Se presenta una propuesta para la interrelación de distintos enfoques disciplinarios con el fin de abordar fenómenos y problemas sociales. Se específica la manera en cómo algunos elementos de diversas disciplinas entran en contacto con los fenómenos y problemas sociales; seguido de ello, se señalan los supuestos que dan sentido a dicha propuesta; y, finalmente, se indica cuál es el papel que pueden desempeñar las disciplinas y cómo se articulan éstas entre sí para dar cobertura a dichos fenómenos y problemas. En los comentarios finales se apuntan algunas líneas de investigación de interrelación disciplinaria que se pueden desarrollar a partir de la presente propuesta.

Palabras ClaVe: Interdisciplina, Multidisciplina, Transdisciplina, Ciencia básica, Ciencia aplicada.

\section{ABSTRACT:}

In this paper presents a proposal for the interrelation of different disciplinary approaches to address social phenomena and problems. Pointing out how this phenomena and problems are linked to some elements of different scientific disciplines; after that, the assumptions that give meaning to said proposal are indicated; and, finally, it is stressed what is the role that the other disciplines can play and how they are articulated among themselves to cover the question asked or the defined problem. In the final comments, some lines of disciplinary interrelation research that are being developed based on the present proposal are pointed out. KEYWORDS: Interdisciplinary, Multidisciplinary, Transdisciplinary, Basic scienc, Applied science, Basic science.

\section{INTRODUCCIÓN}

Uno de los intereses actuales en la investigación científica ha sido la interrelación y vinculación de dos o varias disciplinas de distinta índole y nivel para el estudio de un fenómeno social y/o de la solución de un problema práctico. Este interés tiene que ver con responder la pregunta respecto a la manera en cómo se podría dar dicha interrelación, así como con encarar las dificultades que conlleva el vincular disciplinas que 
limítrofes entre sí o estar totalmente distanciadas en términos epistemológicos, teóricos, metodológicos o, incluso, de sus referentes empíricos (Kumar, 1995; Follari, 2013).

Cabe señalar que una de las motivaciones que ha alentado el interés antes mencionado ha sido la suposición de que los fenómenos de la vida social son multidimensionales; es decir, que son de naturaleza compleja, compuestos por factores, variables o elementos de distinta índole y dimensión que, en su conjunto, no pueden ser abarcados por una sola disciplina. A partir de dicho supuesto, se ha propuesto que la investigación de un fenómeno y la solución de una problemática en una dinámica social, dada su naturaleza global, requiere de la interconexión, comunicación, organización e interrelación de diferentes disciplinas (Heckhausen, 1985; Wallerstein, 1991).

Así pues, haciendo uso de los conceptos de inter, multi y transdisciplina, se han generado reflexiones y propuestas que intentan responder al problema de relación de dos o más disciplinas para estudiar los fenómenos inmersos en una dinámica social particular, sin perder de vista el término complejidad. Es importante señalar que, aunque es posible encontrar ciertas similitudes entre dichas propuestas, muchas de ellas difieren entre sí en lo referente a la postura que adoptan respecto a cómo debería darse la relación, vínculo y conexión de distintas disciplinas para abordar un fenómeno social específico; asimismo, disienten notoriamente respecto a lo que cada una conceptúa como interdisciplina, multidisciplina o transdisciplina.

Casos ilustrativos de ello lo representan las propuestas teóricas o aplicadas de, por ejemplo, el trabajo de García (2006) respecto al diagnóstico, modificación y evaluación de los problemas de desarrollo agrícola; los estudios demográficos sobre población y desarrollo de Menkes (2008); los propuestas sobre estudios ambientales de Paz (2008), las reflexiones sobre la bioética de González (2008), las propuestas de Rodríguez (2011) respecto del diagnóstico, pronóstico y tratamiento de individuos que han cometido delitos; entre otros. En una revisión de estas propuestas, que difieren en nivel, características y alcances, es posible ubicar diferencias sustanciales respecto de cómo debería ser la relación entre disciplinas.

Todos los trabajos realizados en este sentido han hecho patente la necesidad de desarrollar propuestas científicas que permitan incidir en fenómenos sociales o en problemáticas sociales específicas desde una visión multidimensional. En este contexto, en el presente trabajo se presenta una propuesta para la interrelación de distintos enfoques disciplinarios con el fin de abordar fenómenos sociales en los que el comportamiento humano juega un papel funcional relevante en el planteamiento de problemas de algún fenómeno social o como fuente de solución de algún problema social a resolver. Para dar paso a la descripción de tal propuesta, es necesario esclarecer algunos aspectos importantes que le dan sentido y la fundamentan, los cuales se señalan en los siguientes apartados.

\section{LA MULTIDIMENSIONALIDAD DE LOS FENÓMENOS SOCIALES Y LA ORIENTACIÓN DEL TRABAJO CIENTífICO DEL SIGLO XXI}

En el siglo XVII la revolución científica orientó el trabajo de la ciencia en general hacia el estudio de las relaciones entre elementos, variables o causas para explicar los fenómenos del mundo e, incluso, para incidir en ellos \#remplazando el estudio que se realizaba en la época de los griegos y parte de la Edad Media, el cual consistía en la descripción de las sustancias o "esencias" de los fenómenos\#. Fue así como se instituyó la visión del mundo de la ciencia clásica, dominante durante casi tres siglos, la cual se caracterizaba por ser mecanicista, reduccionista, atomista y basada en el modelo de causalidad lineal; es decir, se veía el mundo como suma de partículas, variables, unidades o partes elementales estudiadas en aislado y cuyo funcionamiento se explicaba a razón de una noción de causa y efecto (García, 2006).

Hacia finales del siglo XIX y principios del XX dicha visión del mundo y la forma de proceder de la ciencia clásica se vio limitada para afrontar y dar respuesta a problemas teóricos y prácticos como, por ejemplo, la fuerza ejercida entre dos cuerpos que no estaban en contacto próximo; la organización de los seres vivos dada la diferenciación de relaciones y funciones de las células en distintos tejidos; la liberación de grandes 
cantidades de energía en máquinas de vapor o eléctricas; la automatización; la computación, entre otros. Como efecto directo de dicha situación, se fue edificando una visión del mundo que contrastaba con la anterior visión de la ciencia, al grado que la reemplazó y se manifestó en diferentes campos independientes, tanto científicos como tecnológicos. Dicha nueva visión del mundo se caracteriza por entender, explicar y tratar a las cosas, eventos o fenómenos como totalidades organizadas de elementos relacionados en interacción dinámica, con una función particular y con un fin específico; esto es, se formalizó una visión de sistemas que fue abriéndose paso a lo largo del siglo XX (Kantor, 1953; Bertalanffy, 1976; Ackoff, 2002). A este respecto García (2006) señala:

Hacia mediados del siglo XX se fue abriendo paso una idea que habría de convertirse en tema central en numerosos dominios de la ciencia y que, en primera aproximación, podria sintetizarse asi: los fenómenos que involucran procesos de evolución y cambio, cualquiera que sea su naturaleza (fisica, quimica, biológica, social), tienen lugar en conjuntos organizados cuyo análisis no es fragmentable en elementos aislados. La organización adquiere, por consiguiente, primacia sobre las relaciones parciales. El énfasis se desplaza de las relaciones a las relaciones entre relaciones (p. 117).

Esta visión de sistemas, que impactó en todas las esferas de la ciencia, dio cabida a finales del siglo pasado, y ya entrados al actual, a la concepción de los fenómenos como complejidades de elementos organizados. Con ello, se inclinó el trabajo científico ya no hacia el estudio de las partes elementales de los fenómenos sociales, económicos, medioambientales, biológicos o de cualquier índole, sino hacia el estudio de su complejidad (Capra, 2003).

El supuesto fundamental de esta visión del mundo, en palabras de Corona y Cortés (2009), es que "el universo no está compuesto por entidades simples e indivisibles regidas por leyes universales e inmutables, sino por capas de complejidad creciente que se traducen en plexos de sistemas emergentes cuyo comportamiento entrañan regularidades específicas y cambiantes" (p. 16). De esta manera, señalan dichos autores; la ciencia en general se ha orientado a fundamentarse en teorías y enfoques desde la perspectiva de la complejidad, destinados al tratamiento de la organización de los fenómenos y problemas vigentes en la ciencia.

En concreto, la complejidad de dichos fenómenos y problemas con los que trabaja la ciencia en general radica en su multidimensionalidad, es decir, cada fenómeno o problema social está compuesto por diversas dimensiones (v.g., económicas, legales, médicas, psicológicas, sociales, pedagógicas, etcétera), las cuales, a su vez, están conformadas por factores, elementos o variables interrelacionadas que corresponden al terreno de estudio de una disciplina en particular. Por ende, el abordaje científico de dicho fenómeno o problema social no le es de competencia a una sola disciplina; por el contrario, es necesario que varias de ellas lo aborden desde su dimensión de análisis y que, además, se interrelacionen, comuniquen y vinculen entre sí. Como señala Morín (1990): "es complejo aquello que no puede resumirse en una palabra maestra, aquello que no puede retrotraerse a una ley, aquello que no puede reducirse a una idea simple" (p. 21).

En este sentido, autores como de Hoyos (2016) señalan que los problemas y fenómenos sociales no pueden ser abarcados por una sola disciplina que ha segmentado analíticamente el mundo para su comprensión. Por ello, este autor enfatiza las diferencias marcadas entre la forma en como opera y los fines que persigue la ciencia de nuestro siglo, en contraste con la ciencia de los siglos anteriores: la ciencia del siglo XIX y gran parte del siglo XX se dirigía a la departamentalización del conocimiento en torno a un problema enmarcado en el contexto del paradigma compartido por una comunidad de científicos bajo criterios de verdad, objetividad y coherencia; por el contrario, la ciencia a finales del siglo XX y lo que va del XXI es esencialmente aplicada, esto es, se inclina hacia fines prácticos de adaptación social del conocimiento bajo criterios de eficacia, producción, eficiencia e innovación enmarcados en el contexto de una problemática social a resolver.

En consecuencia, tanto la complejidad de los fenómenos y problemas sociales dada su multidimensionalidad, así como el carácter de operación y la orientación del trabajo de la ciencia del siglo XXI, dan sentido y justifican la necesidad de que distintas disciplinas se relacionen entre sí para dar cobertura a los fenómenos y problemas sociales. Este aspecto se torna relevante para el presente trabajo, 
pues da fundamento y sentido a la propuesta de interrelación disciplinar que se expondrá líneas abajo. Sin embargo, antes de avanzar, es necesario esclarecer a qué se hace referencia por dicho término (i.e., interrelación disciplinar), que en muchos otros trabajos y propuestas se ha referido bajo los conceptos de interdisciplina, multidisciplina y/o transdisciplina.

\section{SOBRE LA INTERRELACIÓN DE DISTINTOS ENFOQUES DISCIPLINARIOS: LA MULTI, INTER Y TRANSDISCIPLINA}

Kantor $(1953,1978,1990)$ menciona que cada disciplina que compone la matriz cultural de la ciencia se ha desarrollado de manera variable e invariable; es decir, por parafrasearlo de alguna manera, se ha desarrollado en una doble vía: por un lado, esforzándose por definir su identidad y conformar su cuerpo de conocimientos respecto a una dimensión propia del mundo y operando sobre clases de eventos particulares; por otra parte, dado que dicha dimensión forma parte de la misma matriz cultural y del mismo mundo de los que forman parte las dimensiones de estudio de las otras disciplinas, cada una de ellas se ha desarrollado a la par de las demás, de tal manera que persiguen los mismos fines (i.e., explicar y/o transformar la clase de eventos con los que trabaja del mundo) y siguen procesos comparables de observación, análisis, interpretación, síntesis y aplicación, al igual que sus hallazgos y explicaciones se pueden integrar a los de otras disciplinas y no se pueden contradecir entre sí.

A este respecto, García (2006) señala que la integración de las ciencias es uno de los resultados de su desarrollo histórico y no "un resultado de la voluntad y de los acuerdos de un grupo de investigación" (p. 24). Con ello, indica que históricamente las distintas disciplinas no se desarrollaron de manera aislada en sus respectivos campos de conocimiento; antes bien, se fueron desarrollando de manera articulada en un proceso de diferenciación e integración. Diferenciación en el sentido de lograr su autonomía e independencia; integración, porque se desarrollaron vinculadas unas con otras de tal manera que se han ido acoplando al grado de que fenómenos o procesos que entran en el terreno de alguna disciplina se han explicado a partir del campo teórico de otra disciplina (v.g., los casos de la fisicoquímica, bioquímica, psicosociología, etc.).

Luego, es importante reconocer que la interrelación entre distintas disciplinas es una característica propia de su desarrollo histórico; sin embargo, también resulta de importancia no perder de vista, como exponen Chávez, Menkes y Solares (2008), que el momento histórico-social actual y sus respectivas consecuencias ha demandado a las distintas disciplinas que aborden un mismo fenómeno desde su respectivo ángulo de trabajo e incluso que se vinculen entre sí para atender, explicar y transformar cada uno de estos fenómenos y problemáticas.

Esta vinculación de disciplinas es lo que actualmente se ha denominado multidisciplina y, más recientemente, interdisciplina y transdisciplina. Pero, es preciso advertir, la significación que se les otorga a dichos términos no es consensuado por todas las comunidades científicas en general, dada su diversidad de sentidos y connotaciones que se les han proporcionado. Para ilustrar lo anterior, a continuación se presentan, a modo de ejemplo, algunas definiciones en torno a lo que algunos autores de distintas áreas de trabajo y provenientes de distintas disciplinas han definido como multi, inter y/o transdisciplina.

Para comenzar, vale la pena definir qué se entiende por disciplina. Al pensar de Beggs (1999), es uno de los conceptos que tiene una significación de mayor consenso entra las comunidades científicas. Beggs señala que por disciplina se entiende un cuerpo de conocimientos o una rama de la ciencia caracterizada por un contenido aceptado. En consonancia con dicha postura, Peña (2011) apunta que una disciplina es un conjunto de saberes teóricos y metodológicos particulares al interior de un campo de conocimiento alrededor de un objeto de estudio. En ese sentido se pueden encontrar aquellas que tienen como fin el de explicar o entender el mundo (v.g., física, química, antropología, sociología); otras, que son de carácter artístico (v.g., música, escultura, cine); algunas más que se caracterizan por ser humanísticas (v.g., literatura, filosofía, estética); o bien, las que son de carácter mayormente tecnológico (v.g., medicina, odontología, ingenierías). 
Ahora bien, en el caso de los términos multidisciplina, interdisciplina y transdisciplina, pueden ser considerados polisémicos en el sentido de que cada autor, dada su postura, les ha impreso significados particulares; algunas veces compatibles, otras, totalmente disimiles entre sí. A continuación, se presentan algunas definiciones para dar cuenta de ello, comenzando con los términos multidisciplina e interdisciplina.

Jiménez (2008) define la multidisciplina como un trabajo en el que participan diferentes profesionales, mediante la utilización de cuerpos teórico-disciplinarios, para explicar, comprender y solucionar un problema; mientras que define la interdisciplina como un proceso para conformar un nuevo cuerpo teórico disciplinario con objeto de estudio y metodología propios, generado a partir de dos o más disciplinas.

Para Beuchot (2004), la multidisciplina es la convivencia de diversas disciplinas que abordan un fenómeno determinado pero ello no implica que éstas colaboren o se vinculen entre sí; por su parte, por interdisciplina supone que esas disciplinas estudian un fenómeno, pero colaborando entre sí, trabajando en equipo y persiguiendo el fin de encontrar la manera de obtener un resultado entre todas de forma coordinada.

Nicolescu (2004) señala que por multidisciplina se entiende lo que abarca o afecta a varias disciplinas que comparten perspectivas diversas sobre un mismo objeto de investigación, pero que no entraña la interacción entre éstas; así, por interdisciplina se entiende la transferencia de conocimientos y métodos, la cual, añade, puede darse en tres niveles: (i) de aplicación (v.g. la biología aplicada a la medicina); (ii) epistemológico (v.g., la lógica formal aplicada a la filosofía); y (iii) de producción de nuevas disciplinas (v.g., la transferencia de ciertos métodos matemáticos hacia la demografía).

Para García (2006), en la investigación multidisciplinaria se suman los aportes de diferentes investigadores que pertenecen a distintas disciplinas y que colaboran en un proyecto común o una problemática general que puede ser analizada desde distintos ángulos. En lo que toca a la investigación interdisciplinaria, señala que los participantes también pertenecen a diferentes disciplinas, pero su integración comienza desde el principio de la investigación al definir el problema a ser abordado en conjunto, así como los objetivos y preguntas de investigación; para ello, todas las disciplinas parten del mismo enfoque epistemológico y se encuentran en constante coordinación, comunicación, diálogo e intercambio, para arrojar resultados que, en conjunto con todo el equipo de trabajo, se integran en un resultado común.

Ribes (2018), por su cuenta, señala que la multidisciplina es un campo de intersección de dos disciplinas limítrofes empíricamente, es decir, que comparten fenómenos que pueden ser analizados por ambas disciplinas, desde perspectivas teóricas y metodológicas diferentes, y que la relación entre ellas se da en términos de que una plantea los problemas teóricos a resolver, mientras que la otra provee una contribución metodológica para resolver dicha problema; ejemplos de ello los proporcionan la fisicoquímica, la biofísica, la psicosociología, entre otros. En el caso de la interdisciplina, dicho autor señalaque las interdisciplinas son disciplinas que están conformadas por la conjugación, en distintos niveles, del conocimiento de diferentes disciplinas científicas, tecnológicas, formales y de conocimientos prácticos históricamente consolidados; por ejemplo, para este autor, la medicina, pedagogía, administración, las ingenierías, arquitectura, entre otroa, comprenden casos ilustrativos de campos interdisciplinarios.

En lo que respecta a la transdisciplina, es posible encontrar, también, definiciones diversas como, por ejemplo, la de Kumar (1995) quien señala que la investigación transdisciplinaria busca una completa integración teórica y práctica, en la que los participantes trascienden su disciplina de origen para compartir un marco epistemológico amplio y una cierta meta-metodología que les sirve para integrar conceptualmente las orientaciones de sus análisis: los postulados o principios básicos, las perspectivas o enfoques, los procesos metodológicos y los instrumentos conceptuales.

Trostle (2002), por su parte, señala que por transdisciplina se entiende el trabajo de investigación en el que los investigadores trabajan juntos, usando un marco conceptual compartido que reúne teorías, conceptos y enfoques de disciplinas específicas. Mientras que Morin (2004) señala que por transdisciplina se entiende el establecimiento de un dialogo entre disciplinas y más allá de toda disciplina, asumiéndose distintos niveles de 
realidad; así pues, la transdisciplina tiene como ambición la unidad, la unificación en la diferencia, del objeto y del sujeto, tomando en cuenta que el sujeto forma parte de la naturaleza y también del conocimiento.

Peña (2009) señala que transdisciplina se trata de un conjunto articulado de saberes teóricos, empíricos y prácticos sobre una problemática humana concreta y en el que confluyen saberes de distintas disciplinas, pero donde el eje de organización es la problemática específica. En ese sentido, señala que la medicina, la arquitectura, la ingeniería serían ejemplos de transdisciplinas.

Finalmente, Ribes (2018) señala que la transdisciplina son todas aquellas disciplinas de carácter formal, sin objeto de conocimiento empírico que las defina, como sistemas de cálculo, métrica y representación de fenómenos de otros campos disciplinares, científicos y de otro tipo (v.g., la matemática, la lógica, la teoría de sistemas, la cibernética, entre otras).

Como se puede apreciar en las definiciones que han proporcionado los autores mencionados, en algunos casos comparten cierta similitud, en otros parecen ser compatibles, mientras que en otros son totalmente contradictorios. Por ello, en este trabajo se utilizará el concepto de interrelación disciplinaria, con lo que se hará referencia a la interconexión y vínculo entre distintos enfoques disciplinarios para abordar un fenómeno o problema social. Una vez sentados los aspectos hasta aquí esbozados, en los siguientes apartados se desarrollan algunos aspectos que fundamentan la propuesta de este trabajo.

Es posible deducir de las distintas definiciones esbozadas que para hablar de la interrelación de distintos enfoques disciplinarios se debe partir de una postura específica que fundamente la noción y forma particular de entender la manera en la que deben vincularse y conectarse las disciplinas al momento de abordar un fenómeno o problemática. En atención a lo anterior, en seguida se esbozan, grosso modo, los supuestos que dan sentido a la propuesta de este trabajo.

\section{Aspectos FUNDAMENTALES PARA LA INTERRELACión DE DISTINTAS DISCIPLiNAS}

El primer aspecto por considerar tiene que ver con la manera en la que cada disciplina entra en contacto con ciertos aspectos de la vida cotidiana en un grupo social determinado, específicamente en lo que se refiere al comportamiento de los individuos en una dinámica social. A este respecto, Ackoff(2002), a partir de una visión de sistemas, señala que las disciplinas científicas tienen contacto con los fenómenos sociales identificando una problemática relacionada con ellos, de la cual se formule una pregunta de investigación para explicar, comprender y generar conocimiento teórico o metodológico en torno a dicho fenómeno ciencia básica-; o bien, describiendo dicha problemática como un problema social a resolver, para aplicar conocimiento teórico o metodológico y así solucionarlo -ciencia aplicada-. En concordancia con lo anterior, este autor señala que la labor científica se enfoca en el desarrollo de procedimientos más efectivos tanto para responder preguntas como para solucionar problemas.

Relacionado con lo anterior, García (2006) señala que dicho contacto con los fenómenos se da cargado de teoría; es decir, existe un corpus de principios que delimita la manera en como se conciben y abordan los fenómenos. Así pues, una disciplina, como cuerpo organizado de conocimientos teóricos y metodológicos, se enmarca en un conjunto de principios que comprenden el marco epistemológico que delimita los criterios de orientación del trabajo científico y su concepción del mundo. Dicho marco, en consecuencia, influye decisivamente en el "contacto" de los científicos con los fenómenos sociales en términos de su delimitación empírica y la forma de operar sobre ellos. Asimismo, de éste depende la formulación de las preguntas que se buscan resolver o los problemas que se intentan solucionar.

En la Figura 1 se representa gráficamente una síntesis concentrado de los comentarios de Ackoff (2002) y García (2006), en torno al contacto científico con los fenómenos sociales. Como se puede observar en dicha figura, una disciplina científica, caracterizada como un cuerpo organizado de conocimientos teóricometodológicos enmarcados por una perspectiva epistemológica específica, tiene contacto, dado su objeto de estudio, con una problemática de algún fenómeno social multidimensional, de dicho contacto deriva una 
pregunta de investigación o se dimensiona un problema social a resolver en torno a la dimensión de análisis que le compete.

El siguiente aspecto que es pertinente puntualizar tiene que ver precisamente con el corpus de conocimiento que delimita la manera en la que se conciben los fenómenos y del que depende tanto la formulación de la pregunta como la delimitación de un problema social. En este contexto, partimos del supuesto de que la interrelación entre disciplinas depende, en primera instancia, de la identidad epistemológica, teórica y metodológica de una disciplina específica que delimita o formula una pregunta a responder, o bien, dimensiona científicamente una problemática social a resolver. Este punto se torna relevante dado que el panorama científico actual está caracterizado por disciplinas que difieren en términos epistemológicos; más aún, dentro de una misma disciplina existen posiciones teóricas cuyos fundamentos son incompatibles e inconmensurables. Por ello, muchas veces el esfuerzo de interrelación disciplinar se realiza con disciplinas que distan en términos de sus fundamentos y concepciones.

FIGURA 1

Representación del contacto de una disciplina con un fenómeno

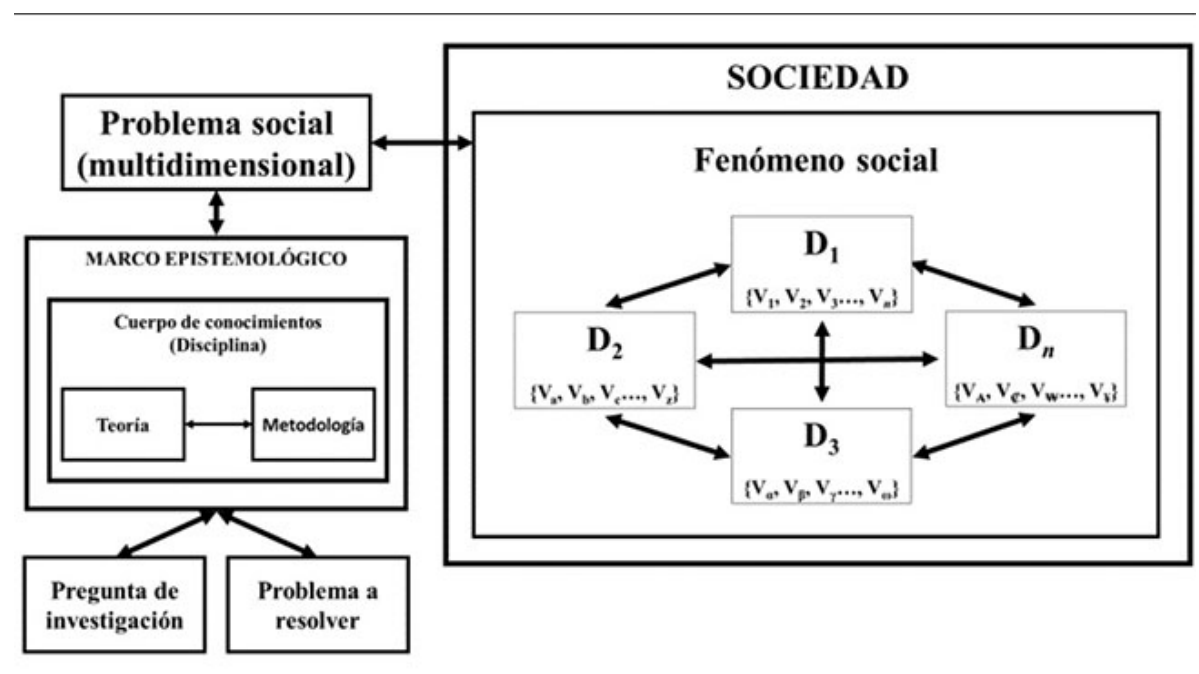

Nota: D1, D2, D3, ..., Dn = Dimensiones del fenómeno social.

$\mathrm{V} 1, \mathrm{~V} 2, \mathrm{~V} 3, \ldots, \mathrm{Vn}=$ Variables pertenecientes a la dimensión $\mathrm{D} 1$

$\mathrm{Va}, \mathrm{Vb}, \mathrm{Vc}, \ldots, \mathrm{Vz}=$ Variables pertenecientes a la dimensión $\mathrm{D} 2$

$\mathrm{V} \alpha, \mathrm{V} \beta, \mathrm{V} \gamma, \ldots, \mathrm{V} \omega=$ Variables pertenecientes a la dimensión $\mathrm{D} 3$

$\mathrm{V} \#, \mathrm{~V} \mathbb{C}, \mathrm{V \#}, \ldots, \mathrm{V} ¥=$ Variables pertenecientes a la dimensión $\mathrm{Dn}$

Fuente: Elaboración propia

Lo anterior está estrechamente vinculado con el tercer aspecto que es necesario considerar en toda interrelación disciplinaria: qué disciplinas se pueden interrelacionar entre sí. Este aspecto es importante debido a que uno de los problemas que ha enfrentado la vinculación de disciplinas es que muchas de ellas distan entre sí en términos de sus características y finalidades (i.e., algunas son mayormente científicas; otras, por ejemplo, tecnológicas). En cuanto a estos dos últimos aspectos, en términos de la solución de una pregunta -ciencia básica-, resulta clave para este trabajo recuperar los señalamientos de Ribes (2018), quien indica que la relación entre distintas disciplinas se da entre campos de conocimiento limítrofes, teórica y/o empíricamente; es decir, que comparten fenómenos que pueden ser analizados por ambos campos desde perspectivas teóricas y metodológicas diferentes. En ese sentido, una disciplina, enmarcada en una noción epistemológica específica, provee el marco teórico que delimita una pregunta a responder sobre un fenómeno, mientras que otra u otras disciplinas colindantes aportan conocimiento y/o métodos o técnicas de investigación empírica para proporcionar elementos que contribuyan a responder a la pregunta planteada.

En lo que respecta a la solución de un problema social -ciencia aplicada-, retomando nuevamente los señalamientos del autor recién citado, la relación entre disciplinas se da por medio del conocimiento aplicable, 
en distintas formas y niveles, en los campos de la vida social por parte de distintas disciplinas. En este caso, las disciplinas que se interrelacionan son aquellas en las que, dada la delimitación y configuración del problema, se identifican elementos del campo social que corresponden a su dimensión de estudio y que juegan un papel funcional destacado como fuente o solución de los problemas que se presentan.

En concordancia con esto último, Rodríguez (2011) señala que "dependiendo del problema concreto que se defina, serán las ciencias o disciplinas concurrentes, entendiendo que hay un número de componentes básicos, mínimo que no se puede rebasar" (p. 16). Asimismo, García (2006) insiste en que el marco epistemológico provee los criterios de configuración del problema social, del que, a su vez, se derivan los objetivos de la investigación y qué disciplinas pueden participar, así como la forma en que lo harán para realizar aportaciones que permitan solucionar el problema.

En atención a los aspectos que se han puntualizado en este apartado, se pueden sintetizar los siguientes puntos que resultan de utilidad para la propuesta de este trabajo:

1. Las disciplinas científicas, con una identidad epistemológica, teórica y metodológica, tienen contacto con los fenómenos sociales al plantearse preguntas a responder (ciencia básica) y/o problemas a resolver (ciencia aplicada).

2. En el marco epistemológico de la disciplina, que delimita tanto las preguntas como los problemas planteados, se definen las propiedades empíricas del fenómeno a ser estudiado.

3. Debido a que muchas de esas propiedades escapan al campo de investigación de la disciplina, otras disciplinas, que pueden compartir o no el marco epistemológico de la disciplina que planteó el problema o la pregunta, colaboran con conocimiento teórico o metodológico para dar respuesta a la pregunta o dar solución al problema planteado desde su dimensión de análisis.

4. Las disciplinas que se pueden interrelacionar para aportar conocimiento teórico o metodológico tienen que ser colindantes con la disciplina que plantea una pregunta a responder o dimensiona un problema a resolver.

5. En el caso de las preguntas a responder las disciplinas colindantes son aquellas que estudian un mismo fenómeno, pero que lo abordan enfocándose en su respectiva dimensión de análisis, a la luz de su particular perspectiva teórica y metodológica.

6. En cuanto a la solución de un problema, las disciplinas colindantes son aquellas en las que algún elemento o elementos identificados en la problemática del campo social le corresponden a su dimensión de trabajo.

Partiendo de estas consideraciones, es fácil suponer que la disciplina que plantea la pregunta o delimita la problemática asume un papel estructurante en la interrelación de las disciplinas correspondientes, de tal manera que su función será la de organizar, coordinar o relacionar dichas disciplinas, así como organizar, evaluar y sintetizar los resultados y conocimientos que aporten las otras disciplinas para resolver la pregunta o solucionar el problema planteado.

En atención a estos puntos, en la Figura 2 se representa gráficamente cómo se da en términos concretos la participación de distintas disciplinas en el abordaje de un fenómeno social complejo. Como se puede observar en dicha figura, una disciplina, con conocimiento teórico-metodológico y una perspectiva epistemológica particular, plantea una pregunta a responder o delimita una problemática social a resolver en el marco de su dimensión de análisis. Las demás disciplinas cuentan también con su propio conocimiento teórico y metodológico derivado de una perspectiva epistemológica específica, que puede o no coincidir con el de la disciplina que plantea la pregunta o el problema; así, dichas disciplinas tienen contacto con una dimensión específica de tal fenómeno. De esta manera, cada disciplina proporciona el conocimiento, métodos, técnicas, estrategias propias para responder la pregunta o resolver el problema que planteó una disciplina en particular.

Así, por ejemplo, en el caso de la ciencia básica la psicología puede proveer un marco que delimite una pregunta a responder de un fenómeno en cualquier ámbito socialmente definido (v.g., educativo, salud, 
medio ambiente, organizacional, forense, entre otros), mientras que otras disciplinas (v.g., la sociología, la antropología, la biología, la economía, la pedagogía, las ciencias penales, entre otras), pueden proveer conocimiento y/o criterios teóricos, así como técnicas y métodos de estudio empírico, para responder a dicha pregunta planteada desde su marco epistemológico. Asimismo, la psicología puede contribuir con conocimiento teórico y/o metodológico para colaborar a responder la pregunta planteada desde otra disciplina en torno a un fenómeno social.

En el caso de la ciencia aplicada, desde la psicología, en su vertiente aplicada, se puede dimensionar científicamente un problema a resolver existente en cualesquiera de los ámbitos sociales de acción profesional donde esta disciplina puede tener inserción y donde la dimensión del comportamiento se conjuga con otras dimensiones, y que, además, puede jugar un papel relevante en la estructuración de dicha problemática. Entonces, frente a problemas sociales de carácter hospitalario, laboral, jurídico, educativo, de medio ambiente, de bienestar social, entre otros, el psicólogo, dado su conocimiento especializado, puede delimitar el problema identificando el papel que juega la dimensión del comportamiento en relación con las otras dimensiones; definir qué otras dimensiones participan en el problema y cómo se relacionan con el factor del comportamiento; e, incluso, analizar si el comportamiento juega un papel destacado en la estructuración de dicho problema social. Los demás profesionales, como médicos, enfermeros, administradores, abogados, criminalistas, pedagogos, ingenieros, entre otros -y según sea el caso-, se interrelacionan con conocimiento práctico con el psicólogo para hacer frente a dicha problemática y resolverla, pero su desempeño profesional es organizado y coordinado por el psicólogo. De igual forma, el psicólogo puede interrelacionarse con conocimiento práctico con otras disciplinas, pero debe ser organizado y coordinado por algún profesional perteneciente a alguna de ellas.

\section{FIGURA 2}

Representación de la interrelación disciplinar para responder una pregunta o resolver un problema Nota: $\mathrm{ME}=$ Marco epistemológico de la disciplina; $\mathrm{T}$ = teoría de dicha disciplina; $\mathrm{y}, \mathrm{M}=$ su metodología. Fuente: Elaboración propia

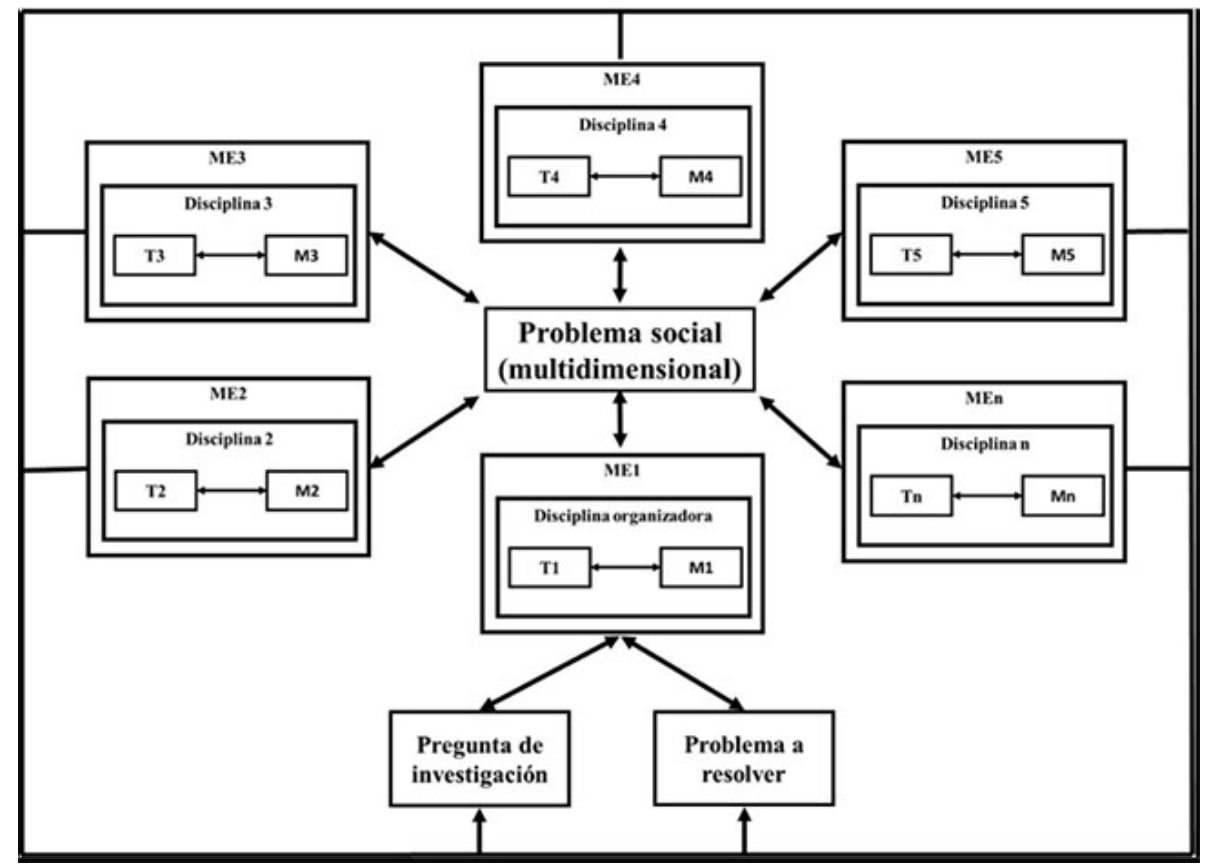

Nota: $\mathrm{ME}=$ Marco epistemológico de la disciplina; $\mathrm{T}$ = teoría de dicha disciplina; $\mathrm{y}, \mathrm{M}=$ su metodología. Fuente: Elaboración propia 


\section{INTERRELACIÓN DISCIPLINARIA PARA EL ABORDAJE CIENTÍFICO DE FENÓMENOS Y PROBLEMAS SOCIALES}

Como se señaló líneas arriba, interrelación disciplinaria es una forma conceptual que se emplea en este trabajo para referirse a la interconexión y vínculo entre distintos enfoques disciplinarios para responder una pregunta o resolver un problema social. Como se apuntó en Reyna, Sánchez, Reyes y Hernández (2017), la interrelación de disciplinas

consiste en una forma de organización de diferentes disciplinas para enfrentar un problema particular [la cual] tiene que ver con la ordenación del conocimiento que se genera a partir de dicha organización [y que] busca la mejor manera de operar en un ámbito determinado para solucionar o analizar un problema, a través de la coordinación de diferentes disciplinas que en menor o mayor medida pueden proporcionar conocimiento, herramientas, o tipos particulares de acción para solucionar un problema característico del ámbito que demande su actuar (p. 37, corchetes nuestros).

Así pues, en el trabajo recién citado se señala que la interrelación de disciplinas tiene como actividad definitoria la organización de disciplinas y la determinación de los elementos que conforman un fenómeno existente en una formación social para definir el problema que será abordado a nivel básico o aplicado, debido a que su finalidad es la de definir cómo operar en un ámbito determinado. Para cumplir con ello, la disciplina convocante define el papel que desempeñarán los profesionales involucrados, los objetivos que se persiguen al abordar científicamente dicho problema definido y los medios para su abordaje, entre otros; donde el sistema social estipula criterios que dan dirección a la organización que debe realizarse para producir conocimiento particular o resultados específicos; y, finalmente, donde la investigación básica se orienta a conocer los lineamientos generales constituyentes del problema social que se aborda; mientras que, en términos de investigación aplicada, se desea conocer las opciones de modificación pertinentes.

Otro aspecto relevante que mencionan Reyna et al. (2017), y que es preciso matizar, es que cuando se interrelacionan diversas disciplinas se hace necesario el empleo de un lenguaje puente, esto es, un lenguaje común, compartido, "producto de la conjunción adaptada de los lenguajes técnicos o tecnológicos de otras disciplinas a las circunstancias que circunscriben el problema socioeconómico con el que se trata" (p. 38). Lo anterior no quiere decir que lo que se pretende es unificar el lenguaje de todas las disciplinas que se interconecten para abordar científicamente un fenómeno. Dicha labor sería difícil de llevar a cabo pues, como se indicó anteriormente, la mayoría de las disciplinas sociales, así como las distintas posturas teóricas dentro de una misma disciplina, parten de principios epistémicos distintos; en consecuencia, el lenguaje técnico propio de cada una de ellas puede ser -y la gran mayoría de las ocasiones es- específico a cada una, por lo que puede ser disímil e incluso incompatible con el de otra u otras disciplinas o perspectivas teóricas. De esta manera, cabe la posibilidad de que las distintas disciplinas o perspectivas teóricas definan un mismo término o concepto con sentidos totalmente disimiles; es decir, que no se refieran a lo mismo a pesar de que todas utilicen la misma palabra. Al respecto, en otro espacio se apunta:

Tener en cuenta lo anterior es sustancial al momento de generar discusiones entre diversas disciplinas o perspectivas teóricas que inciden en términos inter, multi o transdiciplinarios en un campo profesional particular, ya sea para generar aportaciones, contrastar hallazgos, articular propuestas o refutar señalamientos de una u otra perspectiva o disciplina. Cuando lo anterior no se advierte, se generan disputas sin sentido derivadas de la suposición de que todos se refieren a lo mismo al usar un término, cuando en realidad se refieren a cosas o hechos totalmente distintos. La situación se hace más complicada cuando la discusión, a pesar de no tener sentido, se sostiene y se generan nuevas discusiones o nuevas perspectivas en torno a ella (Reyna, 2017, p. 9).

Entonces, cuando se aboga en este trabajo por un lenguaje puente, común o compartido, se hace hincapié en la necesidad de establecer un lenguaje con el que se puedan comunicar los científicos participantes en el abordaje del fenómeno bajo estudio, dada las disimilitudes e incompatibilidades de los lenguajes propios y específicos de cada disciplina o postura. Como señalaba Wiener (1969), la regulación del comportamiento entre personas sólo es posible mediante la transmisión de mensajes entre ellas, es decir, mediante un proceso 
comunicativo; en ese sentido, se hace necesario el establecimiento de un código que permita que tal proceso tenga lugar. En este contexto, Wiener señala que el concepto de código refiere a un conjunto de normas y reglas que permite que un conjunto de señales o símbolos formen un lenguaje aceptado y, en consecuencia, puedan ser entendidos por un grupo humano. En ese sentido, las dificultades de comunicación, cuando no existen limitaciones que impidan la emisión o recepción de mensajes, se deben a que el código no es compartido entre el emisor y el receptor. Esta situación se ve ilustrada con las dificultades de comunicación entre dos disciplinas o posturas que cuentan con un lenguaje específico: no comparten un conjunto de normas o criterios que les permitan entenderse entre sí. En consecuencia, cuando se interrelacionan disciplinas debe establecerse un conjunto de principios (i.e., un código) que especifiquen que un conjunto de sonidos, símbolos o formas conceptuales sean comunes y pueden comprenderse entre los científicos que provienen de diversas disciplinas o parten de posturas distintas, pero que en conjunto participan para el abordaje científico, básico o aplicado, de un fenómeno social.

Así, tomando como fundamento los aspectos que se presentaron en el apartado anterior y los apuntes de Reyna et al. (2017), se puede desarrollar una propuesta de interrelación disciplinar. Por su puesto, ésta adquiere matices diferentes en función de que la interrelación de disciplinas sea en torno a responder una pregunta o a resolver un problema. Por ello se vuelve indispensable considerar la forma de proceder de las disciplinas. Dicha cuestión conduce necesariamente a la consideración de los aspectos metodológicos de la ciencia básica y de la ciencia aplicada, las cuales son distintas entre sí, entre otras cosas, por las finalidades que se persigue en cada una de ellas.

Como lo señalan de Gortari (1983) y Bunge (2012), la metodología del proceder de la ciencia básica o fundamental está encaminada a la producción de conocimiento. Ligándolo con los señalamientos que se han apuntado en este trabajo, al responder una pregunta de investigación persiguen la finalidad de explicar, comprender o profundizar en el estudio de un fenómeno. Continuando con los dos autores, el proceder aplicado persigue fines prácticos, es decir, con miras a la adaptación y/o producción de conocimiento para resolver un problema social mediante la modificación de algún aspecto de éste. En atención a esta distinción, es primordial señalar los matices diferentes que puede adquirir la interrelación de disciplinas en una u otra forma de proceder científico.

Es recomendable también advertir que hablar de metodología supone adentrarse en una discusión respecto de qué es esta, si se trata de una disciplina con estatus propio, qué papel juega en la ciencia, su diferencia con otros términos afines como método, entre muchas otras cuestiones que, de acuerdo con Rodríguez y Rosas (2016), llevan entre 60 y 70 años en vigor y que han sido explicitadas en nuestro país - México - en las diferencias de perspectivas entre Eli de Gortari y Mario Bunge (cf. de Gortari, 1980).

No es la intención de este trabajo describir detalladamente las discusiones imperantes al respecto, que han sido abordadas por distintos autores (v. g. Bunge, 1981, 2012; de Gortari, 1980, 1983; Pérez, 1990; Duval, 2003; Muñoz-Martínez, 2003; de la Garza y Leyva, 2010; Rodríguez y Rosas, 2016; entre muchos otros). En vez de ello, nos limitaremos a describir la forma de proceder de la ciencia básica y la de la aplicada, para poder indicar cómo, en función de dicho proceder, se puede dar la interrelación disciplinar en cada una de ellas.

\subsection{La forma de proceder de la ciencia básica y la interrelación disciplinar para responder una pregunta}

Dado que nuestro interés está puesto en el comportamiento humano inmerso en dinámicas sociales, la perspectiva de la que partimos, en el caso del proceder de la ciencia básica para responder una pregunta, es la que de Gortari (1983) señala como el que ha sido de mayor generalidad en las comunidades científicas, tanto naturales como con matices sociales diferentes. Ha sido descrito para el caso de las ciencias sociales y del comportamiento por distintos autores como Campbell y Katona (1953), Briones (1982), Babbie (1990), 
Kerlinger y Lee (2002), Abello (2009), entre muchos otros. Dicho proceder ha sido el de mayor aceptación en casi todas las esferas de la ciencia en cuanto a forma general de producción de conocimiento, como metodología general de investigación, pero ha adquirido especificaciones metódicas particulares en cada disciplina según sus fines, características y objeto de estudio (de Gortari, 1983). Sin pretensiones de ahondar en dichas diferencias, en términos generales, dicho proceder consiste en lo siguiente:

1. Planteamiento del problema cientifico, que consiste en identificar algún tipo de problemática en torno a la explicación o comprensión de algún fenómeno, en términos de ciertas cuestiones que fundamentan una teoría.

2. Desarrollo del marco teórico conceptual o de referencia, que consiste en la exposición de la teoría que fundamenta la metodología empleada, las observaciones realizadas, la recolección, análisis e interpretación de los datos de la investigación.

3. Especificación de la pregunta de investigación, donde el problema se reduce a ciertos aspectos y relaciones fundamentales, generalmente en forma de pregunta, para poder ser susceptible de ser estudiado.

4. Formulación de hipótesis o supuestos, lo cual comprende una respuesta tentativa a la pregunta de investigación o una suposición preliminar a los resultados de investigación.

5. Definición del o los objetivos de la investigación, que consiste en delimitar las metas que persigue el investigador y que encaminan su proceder al realizar el estudio en cuestión

6. Planeación de la investigación o diseño de la investigación, es decir, la especificación de cómo se llevará a cabo la investigación para cumplir con el objetivo, responder la pregunta y, en su caso, aceptar o rechazar la hipótesis o los supuestos.

7. Aplicación de la investigación, en la que la investigación se pone en marcha de acuerdo con la planeación especificada.

8. Recolección de resultados, para lo que se utiliza alguna técnica de recolección de datos para poder ser interpretados.

9. Análisis e interpretación de resultados, en el que se aplica algún tipo de método y técnica para analizar los datos recolectados y se interpretan a la luz del marco teórico que se especificó con anterioridad.

10. Discusión de los resultados, en la que se contrastan los resultados encontrados con los resultados reportados por otras investigaciones en torno a la misma problemática o fenómeno (explicados de forma resumida en el marco teórico de la investigación).

Entonces, tomando en cuenta esto y los aspectos que se puntualizaron en el apartado anterior respecto de la interrelación disciplinaria, para responder una pregunta de investigación, en torno a un problema asociado a un fenómeno social, en el terreno de la ciencia básica el proceder consistiría en lo siguiente:

1. Un investigador perteneciente a una disciplina identifica un problema en torno a un fenómeno social a partir del cual plantea una pregunta a responder. Dicha pregunta, valga reiterarlo, es formulada a la luz del marco epistemológico y de la postura teórica y metodológica de la disciplina a la que se adscribe le quehacer del investigador en cuestión. De esta manera, la respuesta a la pregunta formulada le permitirá al investigador explicar o comprender el fenómeno mencionado desde su respectiva dimensión de análisis. En el caso que nos ocupa, dicho fenómeno tiene que ver con el actuar de las personas inmersas en alguna dinámica social específica.

2. Se identifican o describen los factores, elementos o variables que conforman la dimensión de análisis que les es propia de estudio, para realizar desde su perspectiva el proceso de investigación correspondiente y describir la manera en cómo éstos se relacionan para explicar dicha dimensión del fenómeno. 
3. En el entendimiento de que los fenómenos son multidimensionales, se identifican qué otras dimensiones juegan un papel relevante para la explicación o comprensión global del fenómeno y qué posibles elementos pueden conformarlos o cómo se pueden relacionar con los elementos de su dimensión de análisis.

4. Se definen qué disciplinas son las encargadas de estudiar dichas dimensiones, las cuales son limítrofes en términos de la colindancia que guardan entre sí en torno a la dimensión de análisis del fenómeno abordado. Entiéndase lo anterior como los límites del sistema.

5. Las otras disciplinas aportan conocimiento teórico, en un doble sentido: ya sea para delimitar los elementos, factores o variables, y relaciones entre éstos, que destacan en su dimensión de análisis el fenómeno abordado, pertinentes a la pregunta de investigación; asimismo, aportan conocimiento teórico para explicar dicho fenómeno.

6. Las otras disciplinas aportan conocimiento metodológico, en el sentido de proporcionar métodos, criterios o técnicas de estudio para realizar el proceso de investigación de dicho fenómeno y, de esta manera, generar conocimiento para su explicación y comprensión.

7. Debido a que la pregunta está delimitada por el marco epistemológico, el investigador define criterios de coherencia y pertinencia de las aportaciones teóricas de otras disciplinas respecto a la consistencia con su marco epistémico.

8. Por ello, el investigador organiza la práctica de los otros investigadores en torno a sus aportaciones teóricas y metodológicas.

9. El investigador establece criterios para organizar el conocimiento proporcionado por las otras disciplinas para sintetizarlo y generar una explicación global del fenómeno.

Así, por ejemplo, un investigador puede especificar en torno a una problemática de salud una pregunta de investigación, en la que se pregunta respecto a los factores que promueven que una persona tenga salud o padezca alguna enfermedad específica. De pertenecer a la disciplina psicológica, dicho investigador delimitará los factores que tengan que ver con su dimensión de análisis: factores conductuales individuales como hábitos y tendencias respecto al cuidado o descuido de su salud, capacidades, valoraciones, actitudes respecto al proceso salud-enfermedad, entre otros.

Empero, dicha dimensión se entrelaza con otras dimensiones distintas a las de la conducta individual, en las que destacan factores, variables o elementos como pueden ser de carácter demográficos (e.g., si vive en una zona urbana, si su vivienda se encuentra lejos de algún hospital, las condiciones de salubridad del espacio físico en el que habita, etc.), económicos (e.g., poder adquisitivo para servicios de salud de calidad, agua potable, alimentación, etc.), sociales (e.g., las prácticas de su grupo social de referencia, ideología de su grupo, etc.), políticos (e.g., reformas o leyes en torno al ámbito de la salud); asimismo, entran en juego aspectos que tiene que ver con características biológicas del individuo (e.g., su estado inmunológico, funcionamiento de los diversos sistemas que componen su organismo, características genéticas, endocrinológicas, inmunitarias, etc.), que, en su conjunto, explican y permiten comprenden que una persona o grupo de personas padezcan de enfermen o gocen de salud.

Ante este panorama, el psicólogo puede identificar o apoyarse en otras disciplinas para definir qué otros elementos entran en juego en el fenómeno que está interesado en explicar. Con ello puede seleccionar qué disciplinas son las correspondientes para aportar conocimiento teórico respecto a las explicaciones de dicho fenómeno desde su dimensión o para identificar qué elementos juegan un papel importante; o bien, qué disciplinas pueden aportar conocimiento metodológico para poder estudiar el fenómeno y generar conocimiento en torno a ello. De esta manera, dicho investigador, organiza a tales disciplinas en torno a su dimensión de análisis para comprender cómo afectan los factores propios de otras dimensiones al comportamiento; o bien, cómo el comportamiento se entrelaza con dichos factores; evalúa la pertinencia y coherencia de las aportaciones en torno a si son consistentes con su marco epistemológico; finalmente, sintetiza dichas aportaciones para generar una explicación global del fenómeno. 


\subsection{La forma de proceder de la ciencia aplicada y la interrelación disciplinar para resolver un problema}

Para el caso del proceder de la ciencia aplicada, si bien en ella también puede haber investigación y guardar las mismas características procedimentales que la investigación de la ciencia básica, en el caso específico de la aplicación o adaptación del conocimiento para solucionar una problemática partimos de las perspectivas planteadas por Carpio, Pacheco, Canales y Flores (1996) y por Rodríguez (2003). Si bien dichas propuestas fueron pensadas exclusivamente para describir el desempeño del psicólogo en campos sociales de acción profesional, consideramos que pueden ser extendidas para describir el desempeño de distintos profesionales provenientes de disciplinas que tienen aplicabilidad e inserción social como, por ejemplo, la administración, la medicina, la antropología social, el trabajo social, criminalística, entre otras.

Así pues, haciendo una síntesis de las propuestas de los autores citados, la forma de proceder en el terreno aplicado para resolver un problema sería el siguiente:

1. Análisis de demandas, es decir, se identifican y puntualizna las demandas que se imponen al científico dado un problema específico a resolver.

2. Detección y formulación de problemas, en la que se dimensiona el problema a resolver en virtud de la identificación de las características del problema en términos de los elementos, factores o variables que lo componen y lo promueven.

3. Detección y formulación de soluciones, en la que se emplean procedimientos para identificar recursos, capacidades, necesidades, etc., vinculados al dilema social que se enfrenta y que dan paso a la formulación de posibles soluciones al problema que se presenta.

4. Definición de estrategias de intervención y criterios de éxito, en la que se especifican las acciones particulares para alcanzar los objetivos planteados. Para ello se seleccionan las estrategias de diagnóstico e intervención y se definen los criterios que permitirán evaluar si los cambios observados en la problemática que se enfrenta son evidencia de éxito o fracaso de la intervención que se pretende poner en marcha.

5. Intervención y desarrollo, que se refiere en general al entrenamiento de capacidades y competencias, que se vincula con el uso de técnicas y procedimientos para la solución de problemas concretos ya existentes.

6. Evaluación y seguimiento, que demanda que los datos o resultados arrojados en la intervención y desarrollo sean valorados como evidencia de cambios significativos que evidencien el éxito o fracaso en la solución de la problemática que se enfrenta.

7. Planeación y prevención, que aluden al diseño de planes y la aplicación de estrategias para evitar la aparición o agudización de cierto tipo de problemas sociales. Finalmente,

8. Investigación, que en este caso puede considerarse investigación tecnológica porque tiene como objetivo redundar en la eficiencia de la aplicación del conocimiento básico y/o de las estrategias, técnicas y/o métodos implementados para resolver la problemática.

Para este caso, la forma en como se interrelacionan las disciplinas guarda similitudes estrechas con las descritas para el caso de la ciencia básica; sin embargo, hay ciertos matices que es necesario especificar:

1. Un profesional, perteneciente a una disciplina específica, se enfrenta a un problema social que demanda solución, por lo que lo dimensiona de acuerdo con su marco epistemológico, definiendo y/o analizando los elementos constitutivos propios de su dimensión de análisis.

2. Aplica el conocimiento práctico al problema que enfrenta para evaluar, diagnosticar e intervenir sobre él desde su dimensión de análisis. 
3. Identifica qué otras dimensiones juegan un papel relevante para la solución del problema que enfrenta, qué posibles elementos pueden conformarlas y cómo se pueden relacionar con los elementos de su dimensión de análisis.

4. Se define qué disciplinas son las encargadas de abordar y trabajar con las dimensiones, y los elementos que las conforman que escapan a su dimensión de análisis.

5. Las otras disciplinas aportan conocimiento, métodos de evaluación, análisis y/o intervención.

6. Debido a que el problema ha sido dimensionado desde el marco epistemológico de una disciplina, el profesional en cuestión define los criterios de coherencia y pertinencia de las aportaciones prácticas de las otras disciplinas.

7. El profesional organiza la práctica de los otros profesionales en términos de las evaluaciones, diagnósticos e intervenciones investigadores en torno a sus aportaciones teóricas y metodológicas.

8. Se establecen criterios para organizar y sintetizar el conocimiento proporcionado por los otros profesionales para generar una evaluación global del problema, en la que se identifican los factores relevantes, que juegan un papel destacado para que el problema se sostenga, y los que, al modificarlos, permiten que éste se resuelva.

9. Derivado de lo anterior, se establecen criterios para el diagnóstico general y de un plan de acción general para la intervención sobre los factores relevantes y, de esta manera, resolver la problemática.

Evidentemente todo esto se realiza de acuerdo con la forma de proceder de la ciencia aplicada que se definió anteriormente. Entonces, continuando con el ejemplo sobre salud-enfermedad que se proporcionó líneas arriba, el psicólogo define, desde su dimensión de análisis, los elementos del comportamiento individual de una persona (o grupo de personas) que entran en juego para que un problema de salud específico se presente. Asimismo, en atención a la multidimensionalidad del fenómeno, analiza qué otras dimensiones resultan relevantes y qué elementos de dichas dimensiones pueden ser pertinentes para evaluar, diagnosticar e intervenir de manera global en el problema. De esta manera, identifica qué otros profesionales pueden participar en el estudio y abordaje de dicho problema, estableciendo criterios para organizar sus prácticas y para evaluar sus aportaciones e integrarlas en un todo coherente. Todo ello lo lleva, finalmente, a una evaluación global que deriva en un diagnóstico global y una posible intervención en el caso, en la que tendrán que colaborar los demás profesionales desde su dimensión pertinente. Por otra parte, dicho psicólogo también puede ser organizado por otros profesionales, con aportaciones en su mayoría metodológicas (métodos de evaluación, análisis e intervención) con respecto al problema que se le enfrenta trabajando desde su dimensión propia de estudio.

\section{Comentarios Finales}

Kantor y Smith (2015) señalan que es inevitable que todas las ciencias se interrelacionen debido a que, aunque están separadas y delimitadas, son cercanas unas con otras debido a que son empresas desarrolladas por personas interesadas en aspectos específicos del mundo total; por ello, agregan, todas las ciencias son en cierto sentido ciencias interdisciplinarias; es decir, están estrechamente vinculadas con otras en el sentido de que aprovechan los recursos de otras ciencias en lo que concierne a sus métodos y postulados teóricos. Así, por ejemplo, la astrofísica aprovecha los recursos de la física, la química, e incluso la psicología; los físicos se apoyan de la química y las matemáticas para solucionar sus problemas; la biología, emplea los hallazgos de la física, química, matemática, psicología; la sociología se apoya en la psicología; entre muchos otros ejemplos.

Parafraseando un poco a los autores citados, es posible sostener que cualquier estudiante que se adentra a formarse en alguna área del conocimiento, inmediatamente puede notar que su disciplina se vincula con otras para resolver las preguntas o problemas que se les enfrenta. En ese sentido, ninguna explicación o intervención sobre el tipo de eventos que con los que trata cualquier disciplina puede ser completa si no se consideran todas 
las dimensiones que le atañen, dado que siempre se trata con eventos complejos de carácter multidimensional. Por ello, se torna sustancial examinar cómo las disciplinas se pueden interrelacionar entre sí.

En breve, la postura que sostenemos es que dicha interrelación disciplinar, en términos de responder una pregunta sobre algún fenómeno social o para resolver un problema socialmente delimitado, se da en términos de proporcionar, desde distintos ángulos de dichos fenómenos y problemas, conocimiento teórico o metodológico, ya sea básico o aplicado, para abordar dicha pregunta o problema que ha sido especificada desde el marco epistemológico de una disciplina particular.

Como se señaló líneas arriba, la interrelación de distintas disciplinas no es un ideal utópico que persiga la ciencia: es una demanda que impone la dinámica social, fuente de preguntas a responder o de problemas a resolver con los que se enfrenta el trabajo científico. En este trabajo, se presentó una propuesta que se espera tenga impacto en las cuestiones en las que el comportamiento humano individual o colectivo, en ámbitos sociales, participa de alguna manera o juega un papel de relevancia para explicarlas e incluso para transformarlas y solucionarlas.

Para finalizar este trabajo, es importante señalar que la serie de comentarios vertidos en él fundamentan, en su conjunto, la aplicación de nuestra propuesta a un caso específico que actualmente se ha convertido en un fenómeno social de interés para las ciencias sociales, así como un problema social de importancia que demanda que éstas aporten conocimiento aplicable para ser solucionado: la transgresión de normas formalmente impuestas, como las normas de los códigos penales o constitucionales de un país o Estado específico (v.g., crímenes y delitos de distintas índole, conductas antisociales y disruptivas, entre otras). Por supuesto, los autores esperamos que sea de utilidad para abordar otros problemáticas y fenómenos multidimensionales; o bien, que cuando menos abone una perspectiva para la discusión imperante en las ciencias sociales respecto al trabajo inter, multi y transdiciplinario.

\section{Agradecimientos}

Los autores agradecen al Consejo Nacional de Ciencia y Tecnología (CONACYT) por el apoyo otorgado para financiar la realización de este artículo. $\mathrm{N}^{\circ}$ de becario 630740 y 630745.

\section{BibLiografía}

Ackoff, R. (2002). El paradigma de Ackoff. Una administración sistémica. México: Limusa Wiley.

Abello, R. (2009). Recomendaciones prácticas para la formulación de proyectos de investigación y desarrollo. Barranquilla: Universidad del Norte.

Babbie, E. R. (1990). The practice of social research. Belmont: Wadsworth.

Beggs, D. (1999). Liberating ecological reason through interdisciplinarity. Metaphilosophy, 30(3), 186-208.

Bertalanffy, L. V. (1976). Teoría General de los Sistemas. Fundamentos, desarrollo, aplicaciones. México: Fondo de Cultura Económica.

Beuchot, M. (2004). La hermenéutica analógica en la multidisciplinariedad de las ciencias humanas. En A. M. Chávez, C. Menkes y B. Solares (Eds.), Ciencias sociales y multidisciplina. Memorias de las VII Jornadas Multidisciplinarias (pp. 33-42). México: Universidad Nacional Autónoma de México.

Bunge, M. (1981). La ciencia, su método y su filosofía. Buenos Aires: Siglo Veintiuno.

Bunge, M. (2012). Filosofía de la tecnología y otros ensayos. Lima: Fondo Editorial, Universidad Inca Garcilaso de la Vega.

Briones, G. (1982). Métodos y técnicas de investigaciones para las ciencias sociales. México: Trillas.

Campbell, A. y Katona, G. (1953). The simple survey: A technique for social-science research. En L. Festinger, y D. Katz (Eds.), Research methods in the behavioral sciences (pp. 23-67). Nueva York: Holt, Rinehart and Winston. 
Capra, F. (2003). Las conexiones ocultas. Implicaciones sociales, medioambientales, económicas y biológicas de una nueva visión del mundo. Barcelona: Anagrama.

Carpio, C., Pacheco, V., Canales, C. y Flores, C. (1996). Comportamiento inteligente y juegos del lenguaje en la enseñanza de la psicología. Acta Comportamentalia, 6(1), 47-60.

Chávez, A. M., Menkes, C. y Solares, B. (2008). Ciencias sociales y multidisciplina. Memorias de las VII jornadas multidisciplinarias. México: Universidad Nacional Autónoma de México.

Corona, J. y Cortes, F. (2009). Complejidad y pensamiento crítico. México: Universidad de Guanajuato.

Follari, R. (2013). Acerca de la interdisciplina: posibilidades y límites. Interdisciplina, 1(1), 111-113.

de Gortari, E. (1983). Metodología general y métodos especiales. Barcelona: Ediciones Océano.

de Gortari, E. (1980). La metodología: una discusión y otros ensayos sobre el método. México: Grijalbo.

de Hoyos, A. (2016). El método científico en la época de la inter y transdisciplina. En A. de Hoyos y G. ML. Riquelme (Coords.), Estudios sobre metodología de la ciencia: su impacto en la teoría y la práctica (pp. 253-268). México: Instituto Politécnico Nacional.

de la Garza, E. y Leyva, G. (2010). Tratado de metodología de las ciencias sociales. México: Fondo de Cultura Económica.

Duval, G. (2003). Ciencias sociales y experimento. En G. Hernández y L. M. Rodríguez (Coords.), Filosofía de la experiencia y ciencia experimental (pp. 59-79). México: Fondo de Cultura Económica.

García, R. (2006). Sistemas complejos. Conceptos, método y fundamentación epistemológica de la investigación interdisciplinaria. Barcelona: Gedisa.

González, J. (2008). La bioética como multidisciplina. En A. M. Chávez, C. Menkes y B. Solares (Eds.), Ciencias sociales y multidisciplina. Memorias de las VIIJornadas Multidisciplinarias (pp. 195-205). México: Universidad Nacional Autónoma de México.

Heckhausen, H. (1985). Disciplina e interdisciplinariedad: problemas de la enseñanza y la investigación en las universidades. México: Asociación Nacional de Universidades e Instituciones de Educación Superior.

Jiménez, M. L. (2008). Ciencias sociales y multidisciplina. En A. M. Chávez, C. Menkes y B. Solares (Eds.), Ciencias sociales y multidisciplina. Memorias de las VII Jornadas Multidisciplinarias (pp. 115-119). México: Universidad Nacional Autónoma de México.

Kantor, J. R. (1953). The logic of the modern science. Chicago: Principia Press.

Kantor, J. R. (1978). Psicología interconductual. Un ejemplo de construcción cientifica sistemática. México: Trillas.

Kantor, J. R. (1990). La evolución cientifica de la psicología. México: Trillas.

Kantor, J. R. y Smith, N. W. (2015). La ciencia de la psicología. Un estudio interconductual. México: Universidad de Guadalajara.

Kerlinger, F. N. y Lee, H. B. (2002). Investigación del comportamiento. México: McGraw-Hill.

Kumar, K. (1995). From posindustrial to posmodern Society. New theories of the contemporary world. Malden: Blackwell Publishers.

Menkes, C. (2008). La multidisciplina en el debate sobre población y desarrollo. En A. M. Chávez, C. Menkes y B. Solares (Eds.), Ciencias sociales y multidisciplina. Memorias de las VII Jornadas Multidisciplinarias (pp. 65-76). México: Universidad Nacional Autónoma de México.

Morin, E. (2004). Introducción al pensamiento complejo. México: Gedisa.

Muñoz-Martínez, E. J. (2003). El método experimental. En G. Hernández y L. M. Rodríguez (Coords.), Filosofía de la experiencia y ciencia experimental (pp. 13-58). México: Fondo de Cultura Económica.

Nicolescu, B. (2004). La transdisciplinariedad manifiesta. México: Gedisa.

Paz, M. F. (2008). Multidisciplina y estudios ambientales. Reflexiones desde nuestra práctica. En A. M. Chávez, C. Menkes y B. Solares (Eds.), Ciencias sociales y multidisciplina. Memorias de las VII Jornadas Multidisciplinarias (pp. 95-106). México: Universidad Nacional Autónoma de México. 
Peña, T. E. (2011). La formación de investigación en psicología. En C. Carpio (Coord.), Investigación, formación y prácticas psicológicas (pp. 1-22). México: Universidad Nacional Autónoma de México - Facultad de Estudios Superiores Iztacala.

Pérez, R. (1990). ¿Existe el método cientifico? México: Fondo de Cultura Económica.

Reyna, W. (2017). Sobre falsos dilemas y confusiones conceptuales en el ámbito educativo: el caso del término "tarea". Revista de Investigación Multidisciplinaria, 4(6), 8-20.

Reyna, W.E., Sánchez, J., Reyes, R. A. y Hernández, M. (2017). Organización y caracterización de las distintas prácticas en Psicología: Matriz Científica Interconductual. En J. J. Irigoyen, K. F., Acuña y M. Jiménez (Coords.), Aportes Conceptuales y Derivaciones Tecnológicas en Psicologia y Educación (pp. 17-42). México: Qartuppi.

Ribes, E. (2018). El estudio cientifico de la conducta individual: Una introducción a la teoría de la psicología. México: Manual Moderno.

Rodríguez, L. (2011). Criminología clínica. México: Porrúa.

Rodríguez, M. L. (2003). La inserción del psicólogo en el campo aplicado. Psicología y Ciencia Social, 5(1), 11-19.

Rodríguez, L. M. y Rosas, C. P. (2016). ¿Qué hacer en metodología de la ciencia? Entre la epistemología y la filosofía de la ciencia. En A. de Hoyos y G. ML. Riquelme (Coords.), Estudios sobre metodología de la ciencia: su impacto en la teoría y la práctica (pp. 39-56). México: Instituto Politécnico Nacional.

Trostle, J. (2002). Algunas reflexiones sobre las ciencias sociales y biomédicas: ¿dos catedrales o un arquetipo inconcebible? En M. Gogna y S. Ramos (Coords.), Experiencias innovadoras en salud reproductiva. La complementación de las ciencias médicas y sociales (pp. 21-28). Buenos Aires: Centro de Estudios de Estado y Sociedad.

Wallerstein, I. (1991), Análisis de los sistemas mundiales, en A. Giddens (Ed.), La teoría social hoy. México: Alianza Editorial.

Wiener, N. (1969). Cibernética y sociedad.Bs. As.: Sudamericana. 